\title{
PULLBACKS AND UNIVERSAL CATENARITY
}

\author{
NoÔmen Jarboui And Ayada Jerbi
}

\begin{abstract}
This paper deals with the universal catenarity of a pullback construction ring. It seeks necessary and sufficient conditions for such a pullback to have the universal catenarity, improving some known results. Its main result leads to new examples of universally catenarian domains.
\end{abstract}

\section{Introduction}

All rings considered in this paper are integral, commutative with unit and with finite Krull dimension. For a domain $R$, we denote by $q f(R)$ its quotient field, $R[n]$ the ring of polynomials in $n$ indeterminates with coefficients in $R$ (for $n=1, R[1]=R[X]$ is the ring of polynomials in one indeterminate), and by $\operatorname{Spec}(R)$ the set of all prime ideals of $R$. The Krull dimension of a ring $R$, written $\operatorname{dim}(R)$ is the supremum of the lengths of chains of prime ideals in $R$. The valuative dimension of $R$ is the supremum in $\mathbf{N} \cup\{+\infty\}$ of the sequence $(\operatorname{dim}(R[n])-n)_{n \in \mathbf{N}}$ (cf. [7]). If $p$ is a prime ideal of $R$ we denote by $h t p$ its height (that is the Krull dimension of $R_{p}$ ) and by $h t_{v} p$ its valuative height (the valuative dimension of $R_{p}$ ).

Recall that an integral domain $R$ of finite Krull dimension $n$ is a Jaffard domain if its valuative dimension, $\operatorname{dim}_{v}(R)$, is also $n$. A locally Jaffard domain is a finite-dimensional domain $R$ such that $R_{p}$ is a Jaffard domain for each $p \in \operatorname{Spec}(R) . R$ is said to be totally Jaffard, if $R / p$ is locally Jaffard for each prime ideal $p$ of $R$. Finite-dimensional Prüfer domains and Noetherian domains are locally Jaffard domains.

2000 Mathematics Subject Classification. Primary: 13B02; Secondary: 13C15 13A17, 13A18, 13B25, 13E05.

Key words. Krull dimension, Prüfer domain, Noetherian domain, strong S-domain, universally catenarian domain, algebraic extension modulo $I$. 
Let $I$ be a nonzero ideal of an integral domain $T, \varphi: T \rightarrow E:=$ $T / I$ the natural projection, and $D$ an integral domain contained in $E$. Then $R=\varphi^{-1}(D)$ is the integral domain arising from the following pullback of canonical homomorphisms:

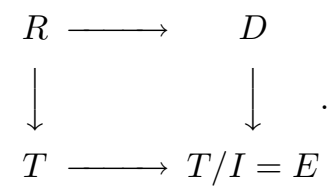

We write $R:=(T, I, D)$ and we shall assume that $D$ is properly contained in $T / I$ and hence, that $R$ is properly contained in $T$. Pullbacks have for many years been an important tool in the arsenal of commutative algebraists because of their use in producing examples. They have become so important that in recent years there have been many papers devoted to ring - and ideal - theoretic properties in pullback domains. The goal of this paper is to determine how the universal catenarity of $D$ and $T$ influence that of $R$.

Our work is motivated by three papers. The first is [2], in which, D. F. Anderson et al. studied universally catenarian domains of $D+M$ type. The second is [12], in which, M. Fontana et al. studied the (universal) catenarity for the $A+X B[X]$ domains. Our third motivation is [4], in which A. Ayache and the first author both developed several techniques useful for studying the S-property in pullbacks in greater generality and derived characterizations of the S-property in any arbitrary pullback.

Recall that a domain $R$ is said to be catenarian if, for each consecutive pair $p \subset q$ of primes in $R, h t q=h t p+1$. The class of catenarian domains is stable under localization and quotient but not under polynomial extensions. Thus in [5], the authors said that a domain $R$ is universally catenarian if $R[n]$ is catenarian for all $n$. Examples of universally catenarian domains are Cohen-Macaullay domains, Prüfer domains, Noetherian domains of (Krull) dimension 1 and finitely generated algebra over a field. We assume familiarity with the above concepts, as in $[\mathbf{1}],[\mathbf{2}],[\mathbf{5}],[\mathbf{1 3}]$.

The main result of this paper is a criterion for the universal catenarity of the pullback $R:=(T, I, D)$, generalizing $[\mathbf{2}$, Theorem 2.2]. It allows us to give new examples of universally catenarian domains, such as the domains $\mathbf{Z}+X \mathbf{Q}[X, Y]$ (where $\mathbf{Z}$ is the ring of integers and $\mathbf{Q}$ the field of rational numbers) and $K+X K[X, Y]$ (where $K$ is an arbitrary field). 
The symbols $T, D, I, R$ have the above meaning throughout the paper. We use " $\subset$ " to denote proper containment and " $\subseteq$ " to denote containment.

Transcendence degrees play an important role in our study: if $R \subset S$ are two domains, we denote by $\operatorname{tr} \cdot \operatorname{deg}[S: R]$ the transcendence degree of the field of fractions of $S$ over that of $R$.

Any unreferenced material is standard, as in [14].

\section{Main Results}

Before stating our main result, we recall from $[\mathbf{1 1}]$ that the extension $R \subset T$ is said to be algebraic modulo $I$ if for every two prime ideals $q, q^{\prime}$ of $T$ such that $I \not \subset q^{\prime}, q^{\prime}+I \subseteq q$ and $h t\left(q \cap R / q^{\prime} \cap R\right)=1$, then $R /(q \cap R) \subset T / q$ is algebraic. The extension $R \subset T$ is said to be universally algebraic modulo $I$ if for every set $\left\{Z_{1}, Z_{2}, \ldots, Z_{n}\right\}$ of indeterminates, the extension $R\left[Z_{1}, Z_{2}, \ldots, Z_{n}\right] \subset T\left[Z_{1}, \ldots, Z_{n}\right]$ is algebraic modulo $I\left[Z_{1}, Z_{2}, \ldots, Z_{n}\right]$.

Recall that a domain $R$ is a strong $S$-domain (strong $S$ in short) if, for each consecutive pair $p \subset q$ of primes in $R$, the extended primes $p[X] \subset q[X]$ are consecutive in $R[X]$. As the catenarity, the S-property is not stable under polynomial extensions, thus a domain $R$ is said to be stably strong $S$ if $R[n]$ is strong $\mathrm{S}$ for any $n$, (cf. [13]). It is well known that if $R[X]$ is catenarian, then $R$ is strong $\mathrm{S}$. Thus universally catenarian domains are stably strong S-domains, hence locally Jaffard.

Recall also that a ring extension $A \subset B$ satisfies the altitude inequality formula (resp., the altitude formula), if for each prime ideal $q$ of $B$, one has: $h t q+$ tr. $\operatorname{deg}[B / q: A /(q \cap A)] \leq h t(q \cap A)+$ tr. $\operatorname{deg}[B: A]$, (resp., $h t q+\operatorname{tr} \cdot \operatorname{deg}[B / q: A /(q \cap A)]=h t(q \cap A)+\operatorname{tr} \cdot \operatorname{deg}[B: A])$.

Note that the extension $R \subset T$ satisfies the altitude formula if and only if for each prime ideal $q$ of $T$ containing $I, h t q+\operatorname{tr} . \operatorname{deg}[T / q$ : $R /(q \cap R)]=h t(q \cap R)$.

We shall use the following notations: We denote by $D_{T}(I)=\{Q \in$ $\operatorname{Spec}(T) \mid I \not \subset Q\}$ and $D_{R}(I)=\{P \in \operatorname{Spec}(R) \mid I \not \subset P\}$. Therefore it follows from $[\mathbf{9}]$ that $D_{T}(I)$ and $D_{R}(I)$ are homeomorphic topological subspaces respectively of $\operatorname{Spec}(T)$ and $\operatorname{Spec}(R)$ equipped with the Zariski topology.

Our main result is the following: 
Theorem 2.1. Assume that $T$ is universally catenarian and that $R \subset T$ satisfies the altitude formula. Then the following statements are equivalent:

(i) $D$ is universally catenarian, the extension $R \subset T$ is universally algebraic modulo $I$ and the following condition holds:

(*) For each $n$ and each prime $P$ of $R[n]$ containing $I[n]$,

$$
h t P=h t I[n]+h t(P / I[n])
$$

(ii) $R$ is universally catenarian.

Before embarking in the proof of this theorem, we note that under condition (i), the domain $R$ is a stably strong $\mathrm{S}$ (cf. [4, Theorem 2.3]). Thus $R$ is locally Jaffard. Hence $h t p[n]=h t p$ for each prime ideal $p$ of $R$. We recall also Cahen's lemma [8, Proposition 4] which plays an important role in this paper. Note that this lemma holds even for polynomial rings since if $R:=(T, I, D)$, then $R[n]:=(T[n], I[n], D[n])$ for each nonzero integer $n$.

Lemma 2.2. Let $R:=(T, I, D)$ and $P_{0} \subset \cdots \subset P_{n}$ a chain of prime ideals in $R$ such that $P_{n}$ is minimal among primes of $R$ containing $I$ and $P_{n-1}$; then this chain lifts in $T$.

Proof of Theorem 2.1: (i) $\Rightarrow$ (ii) Let $P^{\prime} \subset P$ be two consecutive prime ideals in $R[n]$. Our task is to show that $h t P=h t P^{\prime}+1$. We discuss the following cases.

Case 1: $I[n] \subseteq P^{\prime}$. Then $I[n] \subseteq P$. It follows that $P^{\prime} / I[n] \subset P / I[n]$ are consecutive prime ideals in $D[n]$. But $D[n]$ is catenarian, hence $h t(P / I[n])=h t\left(P^{\prime} / I[n]\right)+1$. Therefore $h t P-h t P^{\prime}=[h t I[n]+h t(P / I[n])]-$ $\left[h t I[n]+h t\left(P^{\prime} / I[n]\right)\right]=1$, and hence $h t P=h t P^{\prime}+1$.

Case 2: $I[n] \not \subset P$. Then $I[n] \not \subset P^{\prime}$. The chain $P^{\prime} \subset P$ lifts to $Q^{\prime} \subset Q$ in $T[n]$, moreover $Q^{\prime} \subset Q$ are consecutive because $D_{R[n]}(I[n])$ and $D_{T[n]}(I[n])$ are homeomorphic. Now since $T[n]$ is catenarian, then $h t Q=h t Q^{\prime}+1$. We deduce that $h t P=h t P^{\prime}+1$.

Case 3: $I[n] \not \subset P^{\prime}$ and $I[n] \subseteq P$. As $P$ is minimal containing $I[n]$ and $P^{\prime}$, the chain $P^{\prime} \subset P$ lifts in $T[n]$, to $Q^{\prime} \subset Q$. The ideal $Q$ can be chosen such that $Q^{\prime} \subset Q$ are consecutive. We have $h t Q^{\prime}=h t P^{\prime}$. Thus it will suffice to show that $h t Q=h t P$. Denote by $q=Q \cap T$ and $p=P \cap R$. We get easily $p=q \cap R$ and $I \subseteq q$. Using the fact that $R$ and $T$ are locally 
Jaffard, it follows from the special chain theorem $[\mathbf{6}]$ that:

$$
\begin{aligned}
h t P=h t p[n] & +h t(P / p[n]) \\
& =h t p+h t(P / p[n])=h t p+n-\operatorname{tr} \cdot \operatorname{deg}[R[n] / P: R / p]
\end{aligned}
$$

and

$$
h t Q=h t q[n]+h t(Q / q[n])=h t q+n-\operatorname{tr} \cdot \operatorname{deg}[T[n] / Q: T / q] .
$$

Thus

$$
h t Q-h t P=h t q-h t p+\operatorname{tr} \cdot \operatorname{deg}[R[n] / P: R / p]-\operatorname{tr} \cdot \operatorname{deg}[T[n] / Q: T / q] .
$$

But tr. $\operatorname{deg}[T[n] / Q: R[n] / P]=0$, since the extension $R \subset T$ is universally algebraic modulo $I$. Moreover we have the following diagram of inclusion relations:

It follows that:

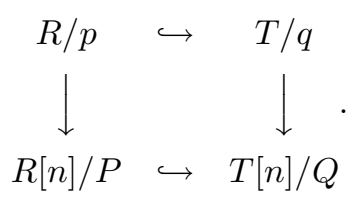

$$
\begin{aligned}
h t Q-h t P= & h t q-h t p+\operatorname{tr} \cdot \operatorname{deg}[R[n] / P: R / p]-\operatorname{tr} \cdot \operatorname{deg}[T[n] / Q: T / q] \\
= & h t q-h t p+\operatorname{tr} \cdot \operatorname{deg}[T[n] / Q: R / p]-\operatorname{tr} \cdot \operatorname{deg}[T[n] / Q: R[n] / P] \\
& -\operatorname{tr} \cdot \operatorname{deg}[T[n] / Q: R / p]+\operatorname{tr} \cdot \operatorname{deg}[T / q: R / p] \\
= & h t q-h t p+\operatorname{tr} \cdot \operatorname{deg}[T / q: R / p]=0 .
\end{aligned}
$$

The latter equality follows from the altitude formula (from the hypothesis). Finally, we get $h t P=h t Q=h t Q^{\prime}+1=h t P^{\prime}+1$, the desired conclusion.

(ii) $\Rightarrow$ (i) Since $R$ is universally catenarian, then so is $D$. Moreover $R$ is stably strong $\mathrm{S}$, hence by [4, Theorem 2.3], $R \subset T$ is universally algebraic modulo $I$. The catenarity of $R[n]$ implies immediately condition $(*)$.

We derive the following results:

Corollary 2.3. Assume that $T$ is a finitely generated $R$-algebra. If $T$ is universally catenarian, then the following statements are equivalent:

(i) $D$ is universally catenarian, the extension $R \subset T$ is universally algebraic modulo I, satisfies the altitude formula and condition (*) is satisfied;

(ii) $R$ is universally catenarian. 
Proof: (i) implies (ii) from Theorem 2.1. Conversely, if $R$ is universally catenarian, it satisfies the altitude formula, that is, every extension $R \subset$ $T$ such that $T$ is a finitely generated $R$-algebra satisfies the altitude formula.

Corollary 2.4. Let $R:=(T, I, K)$ where $K$ is a field. Suppose that $T$ is universally catenarian and for each prime ideal $q$ of $T$ such that $I \subseteq q$, we have:

$$
h t q+\operatorname{tr} \cdot \operatorname{deg}[T / q: R / I]=h t_{R} I,
$$

then the following statements are equivalent:

(i) $R \subset T$ is universally algebraic modulo $I$;

(ii) $R$ is universally catenarian.

Proof: The result follows from Theorem 2.1. Indeed as $R / I \cong K, I$ is a maximal ideal of $R$. Thus, on the one hand, every ideal $P$ of $R[n]$ containing $I[n]$ is such that $P \cap R=I$, and hence, the special chain theorem implies condition $(*)$ of the theorem. On the other hand, each prime ideal $q$ of $T$ containing $I$ is such that $q \cap R=I$, hence condition (1) implies the altitude formula.

Lemma 2.5. If I is a finite intersection of maximal ideals of $T$, condition $(*)$ of Theorem 2.1 is satisfied.

Proof: Denote by $M_{1}, M_{2}, \ldots, M_{m}$ the maximal ideals of $T$ such that $I=\bigcap_{i=1}^{m} M_{i}$. Let $(0)=P_{0} \subset P_{1} \subset \cdots \subset P_{s}=P$ be a chain of prime ideals of $R[n]$ realizing the height of $P$. There exists $1 \leq i \leq s$ such that $P_{i}$ is minimal among the prime ideals of $R[n]$ containing $I[n]$ and $P_{i-1}$. The chain $(0)=P_{0} \subset P_{1} \subset \cdots \subset P_{i}$ lifts in $T[n]$ as $(0)=Q_{0} \subset Q_{1} \subset$ $\cdots \subset Q_{i}$. Thus $Q_{i} \supseteq I$ and hence there exists $1 \leq j \leq m$ such that $Q_{i} \cap T=M_{j}$. It results that $P_{i} \cap R=I$ and by the special chain theorem, $h t P_{i}=h t I[n]+h t\left(P_{i} / I[n]\right)$. Thus $h t P=h t P_{i}+h t\left(P / P_{i}\right)=$ $h t I[n]+h t\left(P_{i} / I[n]\right)+h t\left(P / P_{i}\right) \leq h t I[n]+h t(P / I[n])$, obviously the reverse inequality always hold.

In what follows we recover [2, Theorem 2.2], but first recall from [3] that a ring extension $A \subseteq B$ is said to be residually algebraic if, for each prime ideal $q$ of $B, B / q$ is algebraic over $A /(q \cap A)$. One can check easily that if $R:=(T, I, D)$ and $R \subset T$ is residually algebraic then so is $R[n] \subset T[n]$ for each $n$. Thus $R \subset T$ is universally algebraic modulo $I$ (cf. $[4])$. 
Corollary 2.6. Let $R:=(T, M, D)$, where $M$ is a maximal ideal of $T$. Suppose that $K=T / M$ is the quotient field of $D$. If $T$ and $D$ are universally catenarian, then $R$ is universally catenarian.

Proof: Since $K=q f(D)$, then $D \subset K$ is residually algebraic, hence $R \subset$ $T$ is universally algebraic modulo $M$ (cf. [4]). Localizing at $M, R_{M}$ is the pullback $R_{M}=\left(T_{M}, M T_{M}, K\right)$ thus $R_{M}=T_{M}$, hence, $h t_{T} M=h t_{R} M$. It follows that the extension $R \subset T$ satisfies the altitude formula. On the other hand, it follows from Lemma 2.5 that the extension satisfies condition $(*)$ of Theorem 2.1. Therefore the result follows from the theorem.

Corollary 2.7. If $I$ is a finite intersection of maximal ideals of $T$ and $T$ is a universally catenarian ring. Then the following statements are equivalent:

(i) $D$ is universally catenarian and for each maximal ideal $M$ of $T$ containing $I$, $h t M=h t_{v} I$;

(ii) $D$ is universally catenarian and for each maximal ideal $M$ of $T$ containing $I, T / M$ is algebraic over $R / I$ and $h t M=h t I$;

(iii) $R$ is universally catenarian.

Proof: (i) $\Rightarrow$ (ii) Let $M$ be a maximal ideal of $T$ containing $I$. From the valuative inequality formula for the extension $R \subset T$, we have $h t_{v} M+$ tr. $\operatorname{deg}[T / M: R / I] \leq h t_{v} I+\operatorname{tr} \cdot \operatorname{deg}[T: R]$. Since $h t M=h t_{v} I$, then it follows that $\operatorname{tr} . \operatorname{deg}[T / M: R / I]=0$. Now according to [4, Theorem 2.6], $R$ is a stably strong S-domain so locally Jaffard. Thus $h t_{v} I=h t I$. Consequently $h t M=h t I$.

(ii) $\Rightarrow$ (iii) Under assertion (ii), the ring $R$ is stably strong S. Hence, using [4, Theorem 2.3], the extension $R \subset T$ is universally algebraic modulo $I$. The conclusion follows readily from Theorem 2.1 and Lemma 2.5.

(iii) $\Rightarrow$ (i) We use the same idea as in the proof of $[\mathbf{4}$, Theorem 2.6]. For the sake of completeness, we reproduce this proof with suitable changes at the end. Denote by $M_{1}, M_{2}, \ldots, M_{s}$ the maximal ideals of $T$ such that $I=\bigcap_{i=1}^{s} M_{i}$. Let for instance $M=M_{1}$. Since $M+\bigcap_{i=2}^{s} M_{i}=T$, we can choose $t \in \bigcap_{i=2}^{s} M_{i}$ and $t^{\prime} \in M$ such that $t+t^{\prime}=1$. Set $\mathcal{P}_{1}^{\prime}=((t X-1) T[X]) \cap R[X]$ and $\mathcal{P}_{1}=(M[X]+(t X-1) T[X]) \cap R[X]$. They are not necessarily consecutive, so let $\mathcal{P}^{\prime}$ be maximal among the primes such that $\mathcal{P}_{1}^{\prime} \subseteq \mathcal{P}^{\prime} \subset \mathcal{P}_{1}$ and not containing $I$, and $\mathcal{P}$ be minimal such that $\mathcal{P}_{1}^{\prime} \subseteq \mathcal{P}^{\prime} \subset \mathcal{P} \subseteq \mathcal{P}_{1}$. Thus $\mathcal{P}^{\prime}$ does not contains $I, \mathcal{P}$ contains $I$ and the chain $\mathcal{P}_{1}^{\prime} \subseteq \mathcal{P}^{\prime} \subset \mathcal{P}$ lifts in $T[X]$ as $\mathcal{Q}_{1}^{\prime} \subseteq \mathcal{Q}^{\prime} \subset \mathcal{Q}$. (Note that since $\mathcal{P}^{\prime} \subset \mathcal{P}$ are consecutive, then so are $\mathcal{Q}^{\prime} \subset \mathcal{Q}$.) In fact $\mathcal{Q}_{1}^{\prime}=$ 
$(t X-1) T[X]$ (indeed, as $\mathcal{P}_{1}^{\prime}$ does not contain $I$, it lifts uniquely in $T[X]$ ). So $\mathcal{Q}$ contains $t X-1$, and it contains $I$. It cannot contain any prime containing $t$ (if so, it would contain 1 ). Thus $\mathcal{Q}$ is above $M$. More precisely it is an upper to $M$ (since $t X-1 \notin M[X]$ ). $\mathcal{P}$ is above $I=$ $M \cap R$ in $R[X]$. Let us show it is an upper to $I$. Consider the polynomial

$$
f=(t X-1)\left(t^{\prime} X-1\right)=t t^{\prime} X^{2}-\left(t+t^{\prime}\right) X+1=t t^{\prime} X^{2}-X+1 .
$$

As $t t^{\prime} \in I, f$ belongs to $\mathcal{P}_{1}^{\prime}=((t X-1) T[X]) \cap R[X]$. A fortiori, $f \in \mathcal{P}$, but $f \notin I[X]$, and hence $\mathcal{P}$ is an upper to $I$. Now since $R$ and $T$ are universally catenarian, then $h t \mathcal{P}=h t \mathcal{P}^{\prime}+1$, and $h t \mathcal{Q}=h t \mathcal{Q}^{\prime}+1$. But $h t \mathcal{Q}^{\prime}=h t \mathcal{P}^{\prime}$, thus $h t \mathcal{Q}=h t \mathcal{P}$. Hence $h t M=h t I=h t_{v} I$. The desired conclusion.

We close this section with some new examples of universally catenarian, non Noetherian domains.

Example 2.8. Let $\mathbf{Z}$ be the ring of integers, $\mathbf{Q}$ the field of rational numbers and $X, Y$ two indeterminates over $\mathbf{Q}$. Set $R:=\mathbf{Z}+X \mathbf{Q}[X, Y]:=$ $(T, I, D)$, where $T=\mathbf{Q}[X, Y], I=X \mathbf{Q}[X, Y]$ and $D=\mathbf{Z}$. It is proved in [12, Exemple 3.6], that $R$ is a catenarian totally Jaffard, non Noetherian domain with Krull dimension 3. We improve this result by showing that $R$ is in fact universally catenarian. It follows from [4, Corollary 3.3] that $R$ is a stably strong $\mathrm{S}$-domain. Thus the extension $R \subset T$ is universally algebraic modulo $I$ ([4, Theorem 2.3]), and $D$ and $T$ are universally catenarian. It thus remains to show that the extension $R \subset T$ satisfies first condition $(*)$ of Theorem 2.1 and secondly the altitude formula.

For condition $(*)$, we let $P$ be a prime ideal of $R[n]$ containing $I[n]$ and let $p=P \cap R$. As $R$ is totally Jaffard, we have:

$$
\begin{aligned}
h t P & =h t p[n]+h t(P / p[n]) \\
& \leq h t p+h t(P / I[n])-h t(p[n] / I[n]) \\
& \leq h t p+h t(P / I[n])-h t(p / I) \\
& \leq h t I+h t(P / I[n]) \\
& \leq h t I[n]+h t(P / I[n]),
\end{aligned}
$$

(note that $R$ is catenarian, from [12, Exemple 3.6]). As the reverse inequality always holds, we thus have

$$
h t P=h t I[n]+h t(P / I[n]) .
$$


For the altitude formula, we let $q$ be a prime ideal of $T$ containing $I$.

- If $q=I$, then

$$
\begin{aligned}
h t_{T} q+\operatorname{tr} \cdot \operatorname{deg}[T / I: R / I] & \\
& =h t_{T} X \mathbf{Q}[X, Y]+\operatorname{tr} \cdot \operatorname{deg}[\mathbf{Q}[Y]: \mathbf{Z}]=2=h t_{R}(q \cap R) .
\end{aligned}
$$

- If $I \subset q$, we note that $R / I \subset T / I$ satisfies the altitude formula because $\mathbf{Z} \subset \mathbf{Q}$ and $\mathbf{Q} \subset \mathbf{Q}[Y]$ both satisfy the altitude formula. Hence

$$
\begin{aligned}
h t_{T} q+\operatorname{tr} . \operatorname{deg}[T / q: R /(q \cap R)] & =h t_{T} q+\operatorname{tr} \cdot \operatorname{deg}[T / q: R / I] \\
& =h t_{T}(q / I)+h t_{T} I+\operatorname{tr} . \operatorname{deg}[(T / I) /(q / I): R / I] \\
& =h t_{R}(q \cap R / I)+h t_{T} I+\operatorname{tr} \cdot \operatorname{deg}[T / I: R / I] \\
& =h t_{R}(q \cap R)-h t_{R} I+h t_{T} I+1 \\
& =h t_{R}(q \cap R) .
\end{aligned}
$$

We can conclude that $R$ is universally catenarian.

Example 2.9. Let $K$ be a field, $X, Y$ two indeterminates over $K$ and $R:=K+X K[X, Y]$. We show that $R$ is a 2 dimensional universally catenarian domain which is not Noetherian. Indeed: $R:=(T, I, K)$ where $T=K[X, Y]$ and $I=X T$. The Krull dimension of $R$ is $\operatorname{dim} R=$ $\operatorname{dim} K+\operatorname{dim} K[X, Y]=2([\mathbf{1 0}$, Theorem 2.1]). It follows from $[\mathbf{1 2}$, Proposition 1.10] that $R$ is non Noetherian and from [4, Theorem 3.2] that $R$ is a stably strong S-domain. Hence the extension $R \subset T$ is universally algebraic modulo $I$ ([4, Theorem 2.3$])$. The proof that $R$ is universally catenarian uses Corollary 2.4. Thus, letting $q$ be a prime ideal of $T$ containing $I$, one wants to check that it satisfies condition (1) of this corollary.

- If $q=I$, then

$$
h t_{T} q+\operatorname{tr} . \operatorname{deg}[T / I: R / I]=2=h t_{R} I .
$$

- If $I \subset q$, then,

$$
\begin{aligned}
h t_{T} q+\operatorname{tr} \cdot \operatorname{deg}[T / q: R / I] & =h t(q / I)+\operatorname{tr} \cdot \operatorname{deg}[(T / I) /(q / I): R / I]+h t_{T} I \\
& =h t_{R}(0)+\operatorname{tr} \cdot \operatorname{deg}[T / I: R / I]+h t_{T} I=2=h t_{R} I
\end{aligned}
$$

(since the extension $R / I \subset T / I$ satisfies the altitude formula).

It follows from Corollary 2.4 that $R$ is universally catenarian. 
Using the same previous arguments, we can generalize easily this result by showing that if $K$ is a field and $\left\{X_{1}, X_{2}, \ldots, X_{n}\right\}$ are indeterminates over $K$, then the $\operatorname{ring} R=K+X_{1} K\left[X_{1}, X_{2}, \ldots, X_{n}\right]$ is an $n$-dimensional universally catenarian domain.

Acknowledgements. The authors express greatest thanks to the referees for several helpful remarks concerning presentation.

\section{References}

[1] D. F. Anderson, A. Bouvier, D. E. Dobbs, M. Fontana, And S. KabBAJ, On Jaffard domains, Exposition. Math. 6(2) (1988), $145-175$.

[2] D. F. Anderson, D. E. Dobbs, S. Kabbaj, and S. B. Mulay, Universally catenarian domains of $D+M$ type, Proc. Amer. Math. Soc. 104(2) (1988), 378-384.

[3] A. Ayache and A. Jaballah, Residually algebraic pairs of rings, Math. Z. 225(1) (1997), 49-65.

[4] A. Ayache And N. Jarboui, On questions related to stably strong S-domains, J. Algebra 291(1) (2005), 164-170.

[5] A. Bouvier, D. E. Dobbs, and M. Fontana, Universally catenarian integral domains, Adv. in Math. 72(2) (1988), 211-238.

[6] J. W. Brewer, P. R. Montgomery, E. A. Rutter, And W. J. Heinzer, Krull dimension of polynomial rings, in: "Conference on Commutative Algebra" (Univ. Kansas, Lawrence, Kan., 1972), Lecture Notes in Math. 311, Springer, Berlin, 1973, pp. 26-45.

[7] P.-J. Cahen, Construction $B, I, D$ et anneaux localement ou résiduellement de Jaffard, Arch. Math. (Basel) 54(2) (1990), $125-141$.

[8] P.-J. Cahen, Couples d'anneaux partageant un idéal, Arch. Math. (Basel) 51(6) (1988), 505-514.

[9] M. Fontana, Topologically defined classes of commutative rings, Ann. Mat. Pura Appl. (4) 123 (1980), 331-355.

[10] M. Fontana, L. Izelgue, and S. Kabbaj, Krull and valuative dimensions of the $A+X B[X]$ rings, in: "Commutative ring theory" (Fès, 1992), Lecture Notes in Pure and Appl. Math. 153, Dekker, New York, 1994, pp. 111-130.

[11] M. Fontana, L. Izelgue, and S. Kabbaj, Sur quelques propriétés des sous-anneaux de la forme $D+I$ d'un anneau intègre, Comm. Algebra 23(11) (1995), 4189-4210. 
[12] M. Fontana, L. Izelgue, and S. KabbaJ, Quelques propriétés des chaînes d'idéaux dans les anneaux $A+X B[X]$, Comm. Algebra 22(1) (1994), 9-27.

[13] S. KabbaJ, Sur les $S$-domaines forts de Kaplansky, J. Algebra 137(2) (1991), 400-415.

[14] I. Kaplansky, "Commutative rings", Revised edition, The University of Chicago Press, Chicago, Ill.-London, 1974.

Department of Mathematics

Faculty of Sciences

University of Sfax

P. O. Box 802

3018 Sfax

Tunisia

E-mail address: noomenjarboui@yahoo.fr

E-mail address: ayajerbi-math@yahoo.fr

Primera versió rebuda el 16 d'abril de 2007, darrera versió rebuda el 14 de desembre de 2007. 